\title{
Laparoscopic Jejunostomy as the Standard Procedure of Jejunostomy Tube Placement in Upper GI and Laryngeal Cancers: A Case Series
}

\author{
Farzad Vaghef Davari ${ }^{1}$, Mohammad Shirkhoda ${ }^{2,},{ }^{*}$, Amirmohsen Jalaeefar ${ }^{2}$ and Rezvan Hashemi ${ }^{3}$ \\ 1 Assistant Professor of Surgery, Fellowship of Surgical Oncology, Tehran University of Medical Sciences, Sina Trauma and Surgery Research Center, Tehran, Iran \\ ${ }^{2}$ Assistant Professor of Surgery, Fellowship of Surgical Oncology, Tehran University of Medical Sciences, Department of Surgery, Cancer Institute, Tehran, Iran \\ ${ }^{3}$ Assistant Professor, PhD in Nutrition, Department of Geriatric Medicine, Tehran University of Medical Sciences, Tehran, Iran
}

* Corresponding author: Mohammad Shirkhoda, Tehran University of Medical Sciences, Department of Surgery, Cancer Institute, Tehran, Iran. Tel: +989133529627; Email: mshirkhoda@ sina.tums.ac.ir

Received 2021 January 06; Revised 2021 February 01; Accepted 2021 March 17.

\begin{abstract}
Background: Laparoscopic enteral-feeding access is the best option for patients with advanced upper gastrointestinal, oropharyngeal, and laryngeal cancers needing to maintain their caloric intake before surgery or during chemoradiotherapy.

Objectives: Considering the above-mentioned factors and after a comprehensive review of the related studies, a very simple procedure was selected and the current study tried to modify it to devise a standard surgery.

Methods: During a laparoscopic procedure by a cystostomy catheter system, a jejunostomy tube was placed for 14 patients. All the patients had a 2-month follow-up for complications and performance of the feeding system.

Results: Based on the obtained results, there was no internal leak and peritonitis among the subjects. One patient converted to an open procedure due to perforation during the procedure. In three patients, the extraction of the catheter was encountered during the follow-up period and a replacement was required. One case of wound infection and one case of catheter obstruction occurred among the subjects. Catheter obstruction was easily resolved using warm water and pancreatic enzyme irrigation. There was one patient with partial intestinal obstruction who was managed through nonoperative means. No significant bleeding was encountered during the surgery. Conclusion: Laparoscopic jejunostomy with this method is simple and cost-effective and can be performed within an acceptable timeframe with minimum complications. It is the procedure of choice for upper GI and laryngeal cancer patients, those at increased risk of aspiration, and subjects not candidates of percutaneous endoscopic gastrostomy.
\end{abstract}

Keywords: Enteral nutrition, Jejunostomy, Laparoscopy

\section{Background}

Many patients with upper gastrointestinal (GI), laryngeal, and head and neck malignancies are unable to have food orally and consequently need to receive supplementary enteral nutrition before or after surgery or concomitant with chemotherapy or radiotherapy. Most of these cases are not good candidates for percutaneous endoscopic gastrostomy (PEG) due to upper GI obstruction, risk of aspiration during gastric feeding, and future need to replace the esophagus with the gastric conduit; therefore, surgical procedures remain the only option to provide good GI access to nutrition (1).

Traditionally, open jejunostomy tube placement was the standard procedure for the above-mentioned patients before the laparoscopic era. Different laparoscopic methods have been innovated to perform this procedure; however, many of these methods are time-consuming and unacceptably extend the time of operation (2-7). The main purpose of introducing minimally invasive jejunostomy as a standard procedure is to make the procedure as simple and quick as possible because, almost always, these patients are cachectic and can hardly tolerate complex and long procedures. On the other hand, the placement of large-caliber tubes instead of small-size jejunostomy catheters is very important, especially in the setting of home care in rural and underserviced areas with limited access to standard formulas (1).

\section{Objectives}

Considering the above-mentioned factors and after a comprehensive review of the related studies, a very simple procedure was selected and the current study tried to modify it to devise a standard surgery.

\section{Materials and Methods}

This case series was carried out on 14 patients with dysphagia due to upper GI, head and neck, and laryngeal cancers. The exclusion criteria of the study were vital sign instability, severe sepsis, previous major abdominal surgery (due to related adhesion), uncontrolled coagulation abnormalities, and other contraindications for laparoscopic procedures. Before the surgery, some basic information was recorded for each patient, such as age, gender, and primary cancer. The surgery was performed based on the method introduced by Bobowicz et al. (6) with some complementary modifications.

Pre-operative consultations were requested for all the patients, and informed consent was obtained 
from all the cases. Prophylactic antibiotics and anticoagulants were prescribed before the surgery based on standard protocols. The operations were carried out under general anesthesia with the patient in the supine position and both hands abducted and fixed. Firstly, the jejunostomy site was marked at the left side of the abdomen at the level of the umbilicus and lateral to the rectus abdominis muscle. Laparoscopy was initiated by inserting an 11-mm trocar in the right midclavicular line at the level of the umbilicus as the camera port (inserted by open method) and two 5-mm operating trocars in the right upper quadrant and suprapubic area (Figure 1).

After the primary exploration of the abdominal cavity, a point of the jejunum at a distance of $30 \mathrm{~cm}$ from the Treitz ligament was selected and checked to reach the abdominal wall and marked with methylene blue. Four points with a $1-\mathrm{cm}$ distance were marked on the skin around the previously marked jejunostomy point (primary fixation points). Another two points were marked distally to the mentioned point (secondary fixing points) (Figure 1).

Four 37-mm needles with $1 / 4$ curve (curve of needles changed during the surgery based on the thickness of the abdominal wall) attached to 2-0 Prolene string were introduced into the abdominal cavity through each of the aforementioned primary points and after biting the seromuscular layer of the jejunum around the marked points on this organ (Figure 2) were taken back to the skin and held by a small mosquito forceps (without tying). This step was repeated for two secondary points to avoid jejunal rotation around the fixation points. Subsequently, a suprapubic catheter (Suprapubic Catheterization Kit, BARD Biotech, USA) was inserted into the abdominal wall $2 \mathrm{~cm}$ lateral to the jejunostomy site marked on the skin and introduced into the abdominal cavity exactly under the skin marking point (tunneling) (Figure 3). The catheter was inserted into the

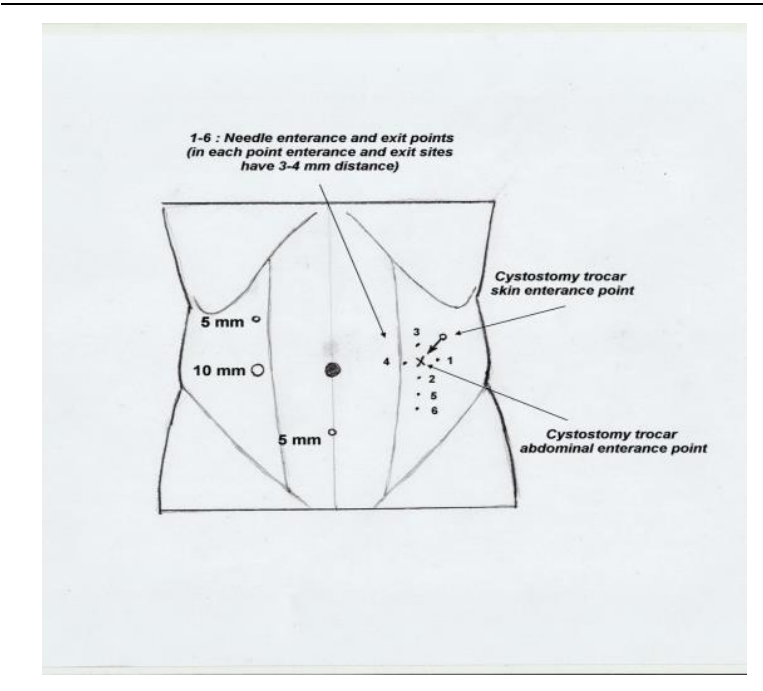

Figure 1. Port sites and cystostomy catheter entrance points on abdominal wall

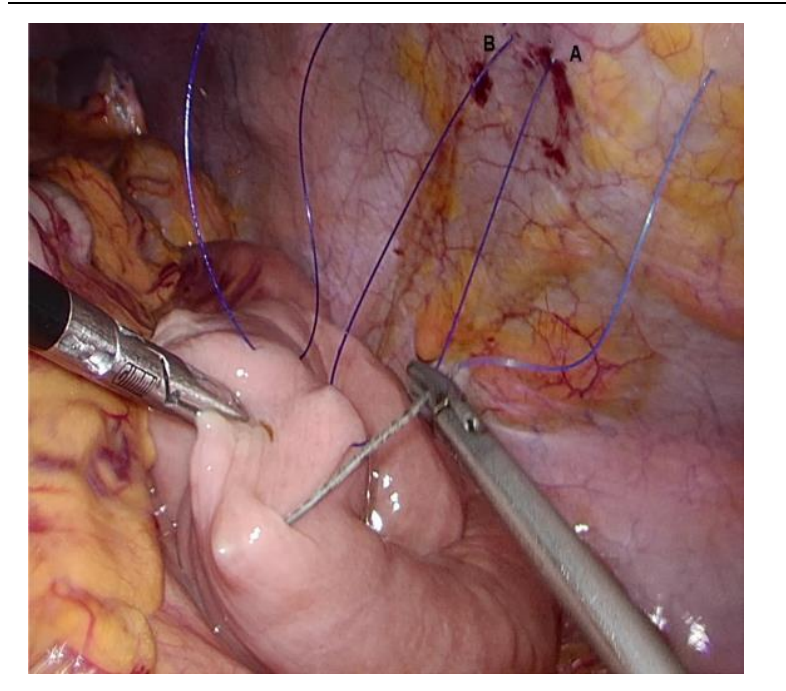

Figure 2. Intra-abdominal view of intestinal hanging procedure: inside view of entrance and exit sites (A and B, respectively) related to point 1 in Figure 1

jejunum through the marked point on the intestine by simultaneously pushing the catheter and upward traction of the four primary strings.

Immediately after inserting the introducer, the trocar was removed, and the $16 \mathrm{~F}$ cystostomy catheter with extra holes was inserted through the sheath at least $10 \mathrm{~cm}$ into the jejunum. The sheath was removed by pulling the tab, the catheter remained in place and was fixed to the skin by sutures. The six hanging strings were also tied over the skin. Normal saline was injected into the catheter, and leakage and tube function were checked by direct observation through the laparoscopic camera. The procedure was terminated by gas desufflation, closing the fascia at the $11-\mathrm{mm}$ trocar site, and skin closure.

During the surgery, other variables, including profuse bleeding in the surgical field, internal leak,

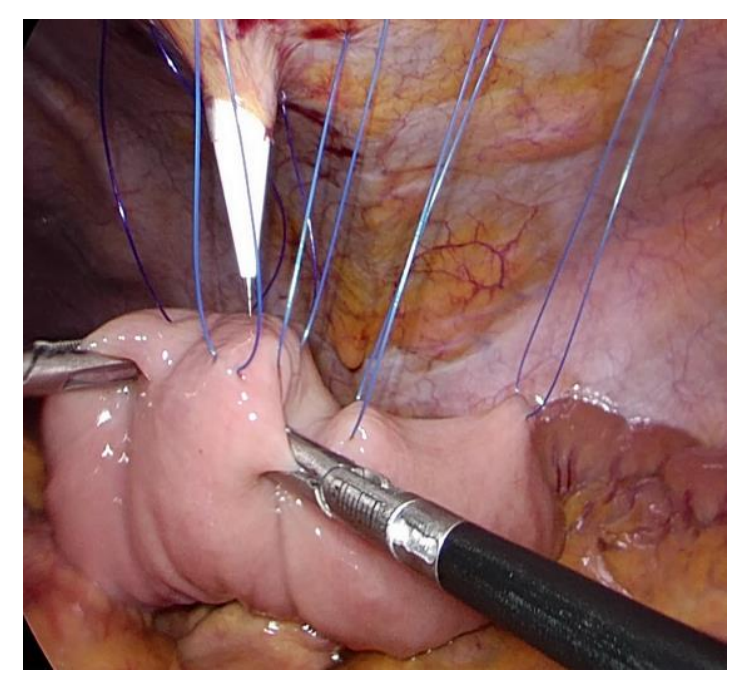

Figure 3. Cystostomy trocar entrance 
and intestinal perforation, were recorded. Feeding through the jejunostomy tube was initiated on the second postoperative day based on the Australian enteral nutrition protocol (8). The patients were discharged from the hospital on the third day and followed up every week for 2 weeks and then every 2 weeks for 2 months after the surgery. The occurrence of peritonitis, wound infection, catheter extraction and obstruction, and intestinal obstruction was recorded during the post-operative visits. The trocar site stitches were removed 1 week and hanging stitches 2 weeks after the surgery, respectively. The data were analyzed using descriptive statistical methods in SPSS software (version 24).

\section{Results}

Among the 14 studied patients, one case withdrew from the study and refused to refer for follow-up after recording some variables; therefore, we included the patient's data only in some parts of the analysis. Out of the 14 patients, 3 cases had an extraction of the catheter, which was replaced with a radiologic guide.

During the surgery, in one of the cases, there was diffuse intestinal edema and consequently difficulty in handling the jejunum. In this particular case, the cystostomy trocar perforated the counterpart of the intestine and we were compelled to convert the procedure to an open type and repair the jejunum. During the postoperative period, there was no procedure-related problem in this case; however, finally, the patient died of a cerebrovascular accident. One patient had pericatheter wound infection that was easily controlled by antibiotics and wound care.

Another minor complication in one patient was catheter obstruction that was resolved using warm water and pancreatic enzymes plus bicarbonate irrigation. One of the patients had a partial intestinal obstruction that was resolved by conservative management. There was no internal leak, peritonitis, or bleeding among the cases during and after the operation. The mean time of operation was reported as $64.2 \pm 13.2 \mathrm{~min}$. All the patients tolerated the enteral feeding regimen based on the aforementioned protocol.

\section{Discussion}

The first laparoscopic jejunostomy technique was introduced in 1990 by O'Regan and Scarrow (9). Since then, many authors have introduced various methods and modifications of this procedure (2-7). Many of the authors used needle catheter jejunostomy techniques requiring special commercial kits with small catheters and a limited amount of nutritional delivery (10), which may not be practical in resource-limited and home care conditions. Others have used laparoscopic or laparoscopy-assisted larger catheter placement methods (2). Laparoscopyassisted procedures use a mini-laparotomy to take out the jejunum and insert the tube (2), which seems to add no benefit to the total laparoscopic techniques in the authors' view. Most surgeons have preferred to utilize total laparoscopy for catheter placement due to the simplicity and less-invasive nature of the operation.

In the era of trocar placement, at least three ports are needed to provide good access to the jejunum; some authors used more trocars which seems unreasonable (5). The authors in the current study were inspired to adopt the procedure by Bobowicz et al. (three trocars) (6); however, it was decided to place the camera and left-hand operator trocars more laterally to have better access to the jejunum and perform simultaneous diagnostic laparoscopy easily if needed.

Jejunal hanging from the abdominal wall has various methods most of which use transabdominal suture application or T-Fasteners. In the present study, the former was selected due to the technical availability and effectiveness. Enterotomy and catheter placement has been performed by various techniques and modifications. Some authors have used purse-string sutures (5), and others have only inserted the catheter through the jejunal wall (6). Some surgeons prefer to use balloon catheters to secure the enterotomy site (2). There seems to be no difference in the complications among the aforementioned methods; therefore, in the present study, a cystostomy catheter was used that cut and entered the jejunal wall directly without making an unwanted wide hole during the insertion. Consequently, there was no need to use purse-string sutures.

As the results of the present study showed, the mean time of operation was about $1 \mathrm{~h}$, which is in line with the findings of other studies $(5,6)$ in this field. There was only one major complication (perforation) requiring converting the procedure to an open type. In a systematic review of this procedure, the frequency of perforation was reported to be 5 in 384 cases (2). This finding is not consistent with the result of the present study due to the small number of patients in the current study and considering the issue that one patient had intra-abdominal edema and adhesions making him susceptible to inadvertent perforation. There were no other major complications, as they have been very rare in other similar studies.

In the present case series, we encountered three cases with catheter dislodgment during the follow-up that was easily fixed. The prevalence rates of catheter dislodgement have been reported to be $4 \%$ in a systematic review (2) and $0.9 \%$ in a study by BenDavid et al. (5). The main reason for this problem is the rupture of the fixating sutures that fix the 
catheter to the skin requiring regular follow-up and re-fixation if needed. This is the reason for the use of nylon string with better tolerance. In the current study, most of the patients were from rural underserviced areas and referred with delay after catheter dislodgement.

The main innovation of the present study was the intramural tunneling of the catheter that prevents loosening of the catheter and external leak around that. There was a wound infection around the catheter in our case series that is in line with other results (5\% in a systematic review) (2) and was easily controlled with antibiotics and wound care.

The prevalence rates of tube clogging were reported to be $2.2 \%$ in one study (5) and $1.6 \%$ in a systematic review (2). In the current study, this problem was easily resolved with catheter injection of warm water and aforementioned pancreatic enzyme solution. There was one case of partial obstruction among the patients of the present study that is consistent with the findings of other studies (2). There were no other major or minor complications (e.g., bleeding, peritonitis, intraabdominal leak, and intestinal obstruction) and no procedure-related mortalities among the patients.

The total complication rate has been reported to be within the range of $0-26 \%$ in various studies with $1.8 \%$ for major complications requiring re-operation (2), and all of them are in line with the results of the current study. We should emphasize that one of the main advantages of this technique is the possibility of simultaneous exploratory laparoscopy that is very useful in some stages of the diseases.

\section{Conclusion}

In conclusion, laparoscopic jejunostomy has been proven to be a fast, minimally invasive, safe, and cost-effective procedure for those patients requiring feeding by enteral access. Complementary modifications have made laparoscopic jejunostomy even simpler than before; therefore, this method can be considered to be the procedure of choice for the placement of enteral feeding access.

\section{Acknowledgments}

This study was approved by the Ethics Committee of Tehran University of Medical Sciences, Tehran, Iran (ID no.: IR.TUMS.MEDICINE.REC.1397.241). The authors would like to express their gratitude to Tehran University of Medical Sciences.

\section{Footnotes}

Author's contributions: Dr. Farzad Vaghef Davari:
Design of the study, performing the operations, and manuscript writing

Dr. Mohammad Shirkhoda: Design of the study and performing the operations

Dr. Amirmohsen Jalaeefar: Performing the operations and manuscript writing

Dr. Rezvan Hashemi: Designing nutritional program and manuscript writing

Ethical Approval: This study was approved by Tehran University of Medical Sciences (School of Medicine Ethics Committee No.: IR.TUMS.MEDICINE. REC.1397.241)

Informed Consent: Informed consent was obtained from all the patients.

Funding/Support: The current study was funded by Tehran University of Medical Sciences (grant no.: 9701-30-38009).

Conflicts of Interest: The authors declare that there is no conflict of interest.

\section{References}

1. Smink DS. Schwartz's principles of surgery. New York: McGraw-Hill; 2015. doi: 10.1097/SLA.0000000000001107.

2. Han-Geurts IJ, Lim A, Stijnen T, Bonjer HJ. Laparoscopic feeding jejunostomy: a systematic review. Surg Endosc. 2005;19(7):9517. doi: 10.1007/s00464-003-2187-7. [PubMed: 15920697].

3. Liu YY, Liao CH, Chen CC, Tsai CY, Liu KH, Wang SY, et al. Single-incision laparoscopic-assisted jejunostomy tube placement. J Laparoendosc Adv Surg Tech A. 2014;24(1):22-7. doi: 10.1089/lap.2013.0360. [PubMed: 24328508].

4. Esposito C, Alicchio F, Escolino M, Ascione G, Settimi A. Laparoscopy-assisted jejunostomy in neurological patients with chronic malnutrition and GERD. Technical considerations and analysis of the results. Pediatr Med Chir. 2013;35(3):1259. doi: 10.4081/pmc.2013.45. [PubMed: 23947112].

5. Ben-David K, Kim T, Caban AM, Rossidis G, Rodriguez SS, Hochwald SN. Pre-therapy laparoscopic feeding jejunostomy is safe and effective in patients undergoing minimally invasive esophagectomy for cancer. J Gastrointest Surg. 2013;17(8):13528. doi: 10.1007/s11605-013-2231-4. [PubMed: 23709367].

6. Bobowicz M, Makarewicz W, Polec T, Kopiejć A, Jastrzębski T, Zieliński J, et al. Totally laparoscopic feeding jejunostomy - a technique modification. Wideochir Inne Tech Maloinwazyjne. 2011;6(4):256-60. doi: 10.5114/wiitm.2011.26262. [PubMed: 23255990].

7. Jimenez Rodriguez RM, Lee MR, Pigazzi A. Trocar guided laparoscopic feeding jejunostomy: a simple new technique. Surg Laparosc Endosc Percutan Tech. 2012;22(5):e250-3. doi: 10.1097/SLE.0b013e31826366ff. [PubMed: 23047399].

8. Dietitians Association. Enteral nutrition manual for adults in health care facilities. Australia: Nutrition Support Interest Group; 2015.

9. O'Regan PJ, Scarrow GD. Laparoscopic jejunostomy. Endoscopy. 1990;22(1):39-40. doi: 10.1055/s-2007-1012785. [PubMed: 2137776].

10. 10- Han-Geurts IJ, Hop WC, Verhoef C, Tran KT, Tilanus HW. Randomized clinical trial comparing feeding jejunostomy with nasoduodenal tube placement in patients undergoing oesophagectomy. $B r J$ Surg. 2007;94(1):31-5. doi: 10.1002/bjs.5283. [PubMed: $17117432]$. 